\title{
LA FILOSOFIA PRIMERA DE EDMUND HUSSERL EN TORNO A 1900
}

Miguel García-Baró

UnIVERSidAD COMPLUTRNSE

MADRID

\section{$\S 1$ Algunos principios}

El acceso más directo a nuestro tema lo proporciona, seguramente, la interpretación del capítulo primero de la Quinta Investigación Lógica, tal como fue impreso en su primera edición. ${ }^{1}$

Va precedido de una importante Introducción ${ }^{2}$ a toda la Investigación Quinta, en cuyas primeras líneas está expresada la verdadera razón por la que a los Prolegómenos a la lógica pura ${ }^{3}$ debían seguir investigaciones fenomenológicas: "Como a todas las unidades ideales, también a las significaciones les corresponden posibilidades reales y, eventualmente, realidades efectivas: a las significaciones in specie les corresponden los actos de significar, y no son aquéllas otra cosa que los caracteres de acto de éstos, captados idealmente." 4

Este breve texto contiene un conjunto de tesis filosóficas fundamentales que la traducción castellana encubre antes que resalta. Es sorpren-

1 Cfr. Edmund Husserl, Logische Untersuchungen, II. Bd., I. Teil (Husserliana XIX/1) (ed. U. Panzer). The Hague/Boston/Lancaster, Martinus Nijhoff, 1984, 352376. (Como se sabe, los puntos en que la primera edición [A] se separa de la segunda [B] van en notas a pie de página.) Recuérdese que A data de 1901, y B, de 1913 -en el caso de la Investigación Quinta.

Entiendanse todas las citas de esta obra referidas a la edición aquí consignada. Pero, para facilitar la búsqueda de los textos, el número de página será el de A (que, como el de B, figura al margen de Hua. XIX/l).

2 A 322-324.

3 Cfr. Hua. XVIII (ed. E. Holenstein) (Den Haag 1975).

4 A 322 . B 343 varía el final: “... no son aquéllas otra cosa que 'momentos' de [aus, no el artículo en genitivo que trae A] éstos, captados idealmente". Es muy interesante observar que, por lo tanto, $B$ no corrige sustancialmente aquí a $A$, pero intenta quitarle su fuerza original. Lo primero hubiera sido tanto como alterar algo esencial del libro primitivo.

En cuanto a la evolución de las ideas de Husserl en este punto tan importante, he señalado algo en mi Fundamentos de la critica de la razón lógica. Ensayo fenomenológico (Universidad Complutense de Madrid 1983), 409-413; pero es preciso investigar más detenidamente el problema. 
dente, sin embargo, comprobar en qué medida han sido desconocidas no sólo por los investigadores que usan nuestra lengua, sino por la inmensa mayoría de los estudiosos en general. ${ }^{5} \mathrm{Y}$ ello, tanto más cuanto que Edmund Husserl las afirma explicitamente en muchos otros lugares de su libro; ${ }^{6} \mathrm{y}$, sobre todo, cuando puede probarse que se hallan en la base misma de toda interpretación adecuada de las Investigaciones lógicas. Tales tesis son:

I. Las unidades ideales, o sea, los objetos ideales son todos especies ( $\mathrm{y}$ todas las especies auténticas son unidades ideales). ${ }^{?}$

II. Las significaciones, o sea, los objetos lógicos son todos unidades ideales y, por consiguiente, especies. Luego; tienen bajo sí individuos.

III. Los individuos del género significación están en la conciencia (o, mejor, en las conciencias de los seres racionales), y son, precisamente, ciertas partes de los actos de significar o dar sentido. No estos actos, no estas vivencias intencionales completas, sino su carácter de acto, o sea, aquello que presta más inmediatamente al acto su indole propia y peculiar.

Estas tres proposiciones suponen la verdad de varias otras aún más importantes, a cuya fundamentación se refieren otras partes de las $I n$ vestigaciones lógicas. Me limitaré ahora a explicitarlas. Reservo su discusión para otro lugar. Se hallan en este grupo, por ejemplo:

IV. Hay objetos ideales y hay objetos reales (que, a la vista de $\mathbf{I}$, constituyen el único dominio en el que pueden hallarse los individuos

5 Debemos a Dallas Willard que el problema haya vuelto a estar en el primer plano. Véase su The Paradox of Logical Psychologism: Husserl's Way Out, en: American Philosophical Quarterly 9 (1972). Reproducido en: J. N. Mohanty (ed.), Readings on Edmund Husserl's "Logical Investigations" (The Hague 1977), 43 ss. En el lugar citado en la nota anterior he mostrado la prehistoria del asunto, por lo que hace a Adolf Reinach y Martin Heidegger. También se encuentran allf algunos argumentos acerca de la imposibilidad intrínseca de lo que Husserl dice.

Una minuciosa refutación de un punto muy importante, estrechamente relacionado con el que ahora nos ocupa, se encuentra en mi An Impossible Theory of Essence: On Edmund Husserl's 'Logical Investigations' (Analecta Husserliana, en prensa).

6 Vid. ante todo la Introducción al tomo II de las Investigaciones, $\$ 2$ (A 5-9). Cfr. Inv. V, § 9 (A 345 s.), § 21 (A 392; seine [die des bedeutungsmässigen Wesens des Aktes] ideierende Abstraktion ergibt die Bedeutung in unserem idealen Sinn) (A $395 \mathrm{~s}$ : die Identität "des" Urteils oder "der" Aussage liegt in der identischen Bedeutung, die sich in den mannigfaltigen Einzelakten eben als dieselbe wiederholt und in ihnen durch das bedeutungsmässige Wesen vertreten ist), etc. Cfr. ya Prolegomena, $\$ \S 39$ y 51 .

7 Cfr. Adolf Reinach, Die obersten Regeln der Vernunftschlüsse bei Kant, en: Gesammelte Schriften, hrsg. von seinen Schülern, Halle 1921, 36-55. Cfr. también mi Adolf Reinach o la plenitud de la fenomenologia, en: El Olivo VII/18 (1983), 217-231. 
de las especies; no entremos tampoco en la cuestión decisiva de si todos los objetos reales son o no obligatoriamente individuos bajo especies). El criterio con el que se divide la esferá total del ser en estos dos campos es el tiempo, de modo que lo real es intrínsecamente temporal y lo ideal es esencialmente atemporal. ${ }^{8}$ Quiere esto decir que la existencia actual de un objeto real es por necesidad existencia en el tiempo.

V. La diferencia entre realidad e idealidad es categorial. ${ }^{\circ}$ No sólo ocurre que todo ser es o real o ideal, sino que, además, ninguna propiedad poseída por un objeto real puede ser poseída por uno ideal (y vale también la conversa). Los seres reales y los ideales sólo comparten el predicado 'ser'.

Sin embargo, todo lo dicho no basta aún para explicar suficientemente por qué la "cuestión del origen del concepto de significación y sus especies más importantes" ${ }^{10}$ debe ser abordada fenomenológicamente, o sea, sobre la base de descripciones del contenido de la conciencia. Hay que añadir otra tesis de extraordinario interés, que está explícitamente afirmada también por las Investigaciones lógicas, pero cuya fundamentación, de nuevo, no examinaré inmediatamente. $Y$ es que:

VI. El conocimiento de las especies ha de depender de algún modo del conocimiento de sus individuos (actuales o posibles). El conocimiento adecuado de los objetos ideales está en función de la adecuación del conocimiento de los objetos reales que a aquéllos les corresponden como sus individuos. $O$, dicho de otro modo, las especies se aprehenden por abstracción a partir de los singulares. ${ }^{11}$

Precisamente analizaremos en seguida que los individuos de las significaciones - las significaciones in individuo, si nos atrevemos a usar un término que viene inmediatamente sugerido por la cita con la que comenzamos- son susceptibles de una presentación originaria excepcionalmente adecuada, según cree Husserl. Forman parte, en realidad, del único conjunto de individuos que admite percepción adecuada — pensemos en los caracteres de los actos de significar que suceden en la conciencia propia-. La lógica pura, que es, ciertamente, una ciencia deductiva, nomológica y apriórica, ocupada sólo con el análisis de ciertos

8 Inv. II, \$ 8 (A 123); Prol. A 77.

9 Pról. A 68: Keine denkbare Abstufung vermag zwischen Idealem und Realem Vermittlungen herzustellen.

Io Inv. V, Intr. (A 322).

I1 Cfr. Pról. $\$ \$ 29$ y 39. Únanse los textos citados en la nota 6 con la línea de Inv. V, $\S 17$ (A 376) suprimida en 1913. Cfr. Inv. UI, passim. También: Inv. V1, $\$ 52$. 
sistemas de especies (los argumentos, los juicios o proposiciones, las preguntas, las órdenes, las suposiciones, los conceptos...), desde el punto de vista de la exactitud de que es capaz la abstracción de sus objetos, está, pues, en una situación excepcionalmente favorable. Podrá ser construida con solidez definitiva una vez que la investigación fenomenológica haya captado adecuadamente los individuos en cuestión. Y sus defectos históricos han de ser sobre todo referidos a la deficiencia de loś estudios fenomenológicos que deben responder a la cuestión fundamental del origen del género significación y sus especies.

Mencionemos todavía dos tesis capitales de las Investigaciones lógicas:

VII. Las significaciones son todas unidades atemporales o ideales; pero no todos los objetos ideales son significaciones. ${ }^{12}$

VIII. Los actos de significar (un grupo de los cuales está constituido por los hoy denominados actos de habla) difieren específica, y no gradualmente, de los actos de la sensibilidad.13

En la Introducción a la Investigación Quinta se ofrece además una descripción más precisa de la localización de los individuos del género significación: "lo significativo en los actos singulares ha de encontrarse justo en el carácter de acto, y no en el objeto; ha de encontrarse en aquello que hace del acto una vivencia 'intencional', una vivencia 'dirigida' a objetos". ${ }^{14}$ Así, pues, la investigación fenomenológica que es el complemento gnoseológico esencial de la lógica pura, debe iniciarse en la búsqueda, en el interior de ciertos actos, del soporte primordial de su intencionalidad. $Y$ ello no es posible si antes no se considera en general la estructura de toda vivencia intencional (= de todo acto de conciencia).

No perdamos de vista desde el inicio mismo del trabajo que el conjunto de estas tesis exige, como condición evidènte de su posibilidad, una serie muy amplia de nuevos principios. Iré haciéndolos explícitos cuando el progreso de mi estudio lo requiera inmediatamente. Pero es imprescindible que cite aquí los dos que considero más interesantes:

IX. Debe haber alguna teoría de la participación consistente (y mucho más que esto: verdadera).

$\mathrm{X}$. Tiene asimismo que ser posible ofrecer una teoría satisfactoria sobre el principio de la individuación.

Un corolario de $\mathrm{X}$ es:

12 Inv. I, $\$ 333$ y 35. Cfr. Inv. VI, $\$ 29$.

13 Inv. I, $\S \S 17-19$; Inv. VI, cap. VI.

14 A 323. 
XI. Debe haber alguna mereologia general completa.

\section{§2. Un argumento a propósito de la percepción externa, y algunos principios más}

Como es sabido, Husserl parte de la psicología descriptiva de Franz Brentano. En especial, acepta dos de los principios de ésta: a) que la característica más esencial de los fenómenos psíquicos por excelencia es la intencionalidad; $b$ ) que todos los fenómenos intencionales son representaciones o bien están basados en representaciones. Sin embargo, el asentimiento está condicionado a una aclaración muy ardua de la noción de representación, por una parte; y, por otra, a la expansión del sentido del término 'fenómeno psíquico', de manera que no designe sólo a fenómenos intencionales (pues Husserl cree que hay vivencias no intencionales).

La primera descripción fenomenológica que se encuentra en la Investigación Quinta tiene por fin mostrar:

XII. Que es preciso separar vivencia y objeto - contra el fenomenismo.

XIII. Que las vivencias concretas tienen partes —entre ellas, partes abstractas.

XIV. Que ninguna parte de una vivencia es parte de su objeto (y ninguna parte del objeto lo es de la vivencia referida intencionalmente a él).

Quizá, por razones que se pondrán pronto de manifiesto, habría que emplear aquí palabras más cautas. Al parecer, Husserl piensa limitada la prueba de estas tres conclusiones a entender por 'objeto' una 'parte del mundo' - desgraciadamente, la determinación precisa de lo que esta expresión significa debe, otra vez, reservarse para más adelante.

'Concreto' y 'abstracto' son términos exactamente definidos en la ontología formal expuesta en la Investigación Tercera. El primero es sinónimo de 'independiente', y el segundo lo es de 'no independiente'. Se llaman 'partes concretas' o 'trozos' las que son independientes respecto del todo en el que están de hecho. Se llaman 'partes abstractas' [Momente] las que son no independientes respecto del todo en que están. Los trozos están vinculados en el todo gracias a un "momento" que fundan entre todos ellos, y que es el responsable directo de la integración del todo (de la diferencia entre la pura suma de trozos y el todo inte- 
grado por sus trozos). Los "momentos", en cambio, no precisan ser atados unos con otros, sino que, por su propia naturaleza, se funden en el todo concreto. ${ }^{15}$

Estas breves nociones de mereología general están supuestas en los argumentos y descripciones que examinamos a continuación. Otras muchas cuestiones ontológicas, así como los graves problemas que afectan a la mereología general en multitud de lugares, deben ser dejados al margen, por el momento. ${ }^{16}$

Ahora se vuelve enteramente comprensible la "definición" de las vivencias que ofrece Husserl: "los acontecimientos reales (Wundt dice, con razón: los sucesos) que, cambiando de momento a momento, vinculados y fusionados unos con otros muy variamente, constituyen la unidad real de la conciencia de cada individuo psíquico". ${ }^{17}$

Hechas estas precisiones, analicemos la descripción inicial ${ }^{18}$ a cuyos objetivos me he referido supra (XII-XIV).

Se trata del examen de una "percepción externa". Husserl pide, ante todo, que se distinga bien el objeto percibido, del contenido vivido o consciente. $\mathrm{Y}$, luego, que se aprenda a diferenciar cada una de las partes de la percepción, de modo que al final pueda verse que ninguna de ellas tiene sentido decir que sea una parte del objeto percibido.

A mi parecer, la reconstrucción total del argumento es ésta:

i) Hay ilusiones perceptivas y alucinaciones.

[ii) Alguna vez hemos salido realmente de alguna ilusión perceptiva o alucinación.]

iii) Una ilusión de este tipo consiste en una referencia intencional perceptiva a un objeto que no existe como tal.

iv) Si el objeto no existe, entonces sus propiedades tampoco existen.

v) La diferencia entre percepción correcta y percepción engañosa no es una diferencia descriptiva.

vi) Respecto de una percepción (alucinación o no) es necesariamente posible, mediante descripción directa [percepción interna adecuada], señalar su objeto y las propiedades de éste -porque es de la esencia de una percepción ser intencional.

vii) Puesto que cabe que el objeto y sus propiedades, tratándose de la "percepción externa", no existan, pero no cabe que no exista la percepción externa que es objeto de una percepción interna adecuada, en-

15 Vid. sobre todo, en Inv. IUI, los $\$ \$ 17$ y 21.

16 He empezado a discutirlos en: Acerca del fundamento de la ontología formal: aporía del realismo e imposibilidad del nominalismo, en: J. Pérez Ballestar (ed.), Análisis y sintesis (Universidad de Salamanca 1984), 173-207. Cfr. B. Smith (ed.), Parts and Moments. Studies in Logic and Formal Ontology (München/Wien 1982).

17 Inv. V, $\$ 2$ (A 326).

18 A $326 s$. 
tonces, y dado que, por otra parte, un ser real no puede contener partes que no existan, el objeto "externo" y sus propiedades no son partes de la percepción referida a ellos.

viii) Pero tiene que haber partes reales en la percepción misma que sean accesibles a la descripción y que caractericen a la percepción como 'percepción de tal objeto dotado de tales propiedades' (cfr. vi). [Seguramente necesitaremos una parte real para cada propiedad, y otra, por ejemplo, para la totalidad del objeto como tal. Éste es un problema fundamental y, desde luego, difícil.]

¿Cuáles son las partes de que habla viiii? Para simplificar, pensemos en una percepción puramente visual. El objeto (=el objeto intencional = el contenido intencional; hay que tener mucho cuidado con no dejarse extraviar por esta última expresión) es, por ejemplo, este libro azul que percibo ahora. El libro mismo, con todas las propiedades que yo miento que él posee ya en cuanto objeto de mi percepción actual, y especialmente su color azul, puesto que pueden estar siendo alucinados o soñados, no están entre las partes reales de la vivencia que es objeto de la percepción adecuada [interna]. Pero sí está adecuada y absolutamente dado que mi percepción lo es de este libro con todas estas propiedades. Como no hago más distinción que vivencia-objeto (tesis $\mathrm{XV}$, que no puede identificarse, en absoluto, con XII), deberé decir que:

XVI. Todo lo dado absolutamente, como no es ni el objeto ni parte suya, es la vivencia concreta y las partes concretas y abstractas de ésta. Una descripción fenomenológica es, justamente, aquella que, por atenerse a lo dado absolutamente, no toma en cuenta el objeto, el contenido intencional, sino sólo y exclusivamente la vivencia, el contenido ingrediente en la conciencia. ${ }^{19}$

Vistas así las cosas, comienza a dejarse entender que Husserl señale, en el texto que comento, estas tres partes en el contenido ingrediente o fenomenológico: el "momento" de color, el carácter perceptivo y el fenómeno perceptivo completo [die volle Wahrnehmungserscheinung]. ${ }^{20}$ El "fenómeno" tiene al menos dos partes que aquí se mencionan: la aprehensión objetivadora y el "momento" de color. ${ }^{21}$ Para este último se emplean varios sinónimos: la sensación de color, el "momento" de color subjetivo cualitativamente determinado, el "momento" ingrediente

19 Inv. V, A 387: Für die phänomenologische Betrachtung ist die Gegenständlichkeit selbst nichts; sie ist ja, allgemein zu reden, dem Akte transzendent. Cfr. Inv. V, §§ 11 y 16. 
de color. ${ }^{22}$ Una percepción concreta deberá, pues, poseer al menos dos partes: carácter de percepción y fenómeno. Veamos con pormenor este punto.

El carácter de percepción debe diferenciar a una percepción de cualquier otro acto. Y ello precisamente porque el "fenómeno" puede ser compartido por percepciones y otras vivencias intencionales (el resto de las "intuiciones"). En efecto, los recuerdos y las fantasías, por ejemplo, son "fenómenos" —o, más exactamente, contienen "fenómenos"- del mismo objeto que, en otras oportunidades, es percibido.

Podría objetarse que los "fenómenos" rememorativos, imaginativos y perceptivos muestran en sí mismos ser diferentes. El objeto recordado, imaginado o percibido bien puede ser idénticamente el mismo (de la misma especie y dotado de las mismas propiedades); pero las "sensaciones" quizá son en los tres casos distintas. Quizá haya "sensaciones" sólo propias de la percepción, junto a "sensaciones" sólo propias del recuerdo y "sensaciones" de nuevos tipos, siempre y necesariamente formando parte de "fenómenos" contenidos en vivencias intencionales de especies diferentes. Si ocurriera así, los tipos de "sensaciones" serían el verdadero fundamento de los tipos de "fenómenos", y éstos, a su vez, podrían desempeñar muy bien un papel de importancia en la clasificación de los actos completos (que, en este caso, son los que constituyen el género "intuición sensible").

Estoy convencido de la inconsistencia de esta objeción; ${ }^{23}$ pero éste es sólo lugar apropiado para suscitarla, no para discutirla a fondo. Por ahora baste aludir a la poca verosimilitud que hay en favor de que un recuerdo y una fantasía se diferencien primordialmente por las "sensaciones" en que se fundan. Me adhiero a la opinión de Husserl: los "fenómenos" tienen plena capacidad para hallarse en cualquiera de las clases de la intuición sensible. No hay "fenómenos" privativos de alguna de estas clases.

Más interesante es caer en la cuenta de que tipos separados de intuiciones sensibles comparten características que caen fuera del "fenómeno" - y que, por lo tanto, no pertenecen tampoco al "carácter" de, por ejemplo, la percepción (naturalmente, tomadas siempre las palabras en las acepciones que, siguiendo el texto de Husserl, hemos fijado arriba). Así, las fantasías son referencias intencionales intuitivas y sensibles, como percepciones y recuerdos, pero, a diferencia de las percepciones y los recuerdos, no "mientan" su objeto como realmente existente (= no son "ponentes"). La "posición" (= la mención del objeto como realmente existente) es, en cambio, común a las otras intuiciones sensibles de que

22 Ibid.

23 Cfr. Inv. V, $\S 14$ y. 15. 
venimos hablando. Los "fenómenos" no son ni ponentes ni no ponentes. La posición es cosa del acto concreto en cuanto un todo. $\mathrm{X}$, como es adecuadamente evidente si un acto es o no ponente, dado que -repito- no utilizamos más distinción que acto-objeto, es forzoso decir que la posición o su ausencia son también partes descriptivas de las vivencias intencionales. En este caso, partes del carácter perceptivo y del carácter rememorativo; pero sólo partes de él.

A esta altura, sin embargo, todavía es más importante considerar el fundamento de la distinción introducida por Husserl en el "fenómeno". En sus palabras, dentro del fenómeno del color del objeto hay que separar la "aprehensión objetivante" y la "sensación de color" o "el momento subjetivo de color, cualitativamente determinado".

Esto significa, en primer lugar, que la "sensación" y su "aprehensión objetivante" son heterogéneas. ¿Por qué?

Las razones de Husserl son dos. La primera, no señalada explícitamente en los parágrafos iniciales de la Investigación Quinta es:

XVII. Que las "sensaciones" no son intencionales. (Siendo así que la "aprehensión objetivante" es el soporte básico de toda intencionalidad -o, aún más: el núcleo de la intencionalidad de todo acto.)

La segunda razón en favor de la heterogeneidad de las partes del "fenómeno" debe expresarse así:

XVIII. Que ningún objeto es la mera suma de sus propiedades "sensibles".

Dedicaré en otro lugar más atención a XVII. Veamos primero XVIII. Si algún objeto pudiera ser la mera suma de sus propiedades sensibles, ¿bajo qué condición resultaría ello favorecer la hipótesis de que "sensaciones" y "aprehensiones" pertenecieran a la misma especie? Mentar intuitivamente semejante objeto consistiría tan sólo en "tener" conscientemente colores, formas, tamaño, rugosidad, humedad, calor, sonido, olor, etc. Habría desaparecido la necesidad de sumar a estos datos sensibles o "propiedades sensibles objetivas" ningún otro factor. Pero las propiedades del objeto no son partes de lo dado fenomenológicamente, como ya hemos visto. No están dadas absoluta y adecuadamente. Lo que está adecuadamente dado es ese "tenerlas" intencionalmente; el cual, en el ejemplo hipotético que propongo, no puede ser sino un "fenómeno" integrado únicamente por "sensaciones". La tesis XVIII sólo es premisa para la conclusión "sensación y aprehensión son heterogéneas' bajo el supuesto de que las "sensaciones" sean accesibles a una 
descripción fenomenológica que las encuentre al menos análogas a las propiedades objetivas sensibles (y se vea entonces en èl deber de explicar con un mismo principio el origen de esta analogia y la transcendencia ${ }^{24}$ de las propiedades objetivas, frente a la inmanencia de las "sensaciones"). ¿Cuál es en realidad la relación entre el "momento" subjetivo de color determinado cualitativamente y el color objetivo, el color del objeto?

\section{§3. Fundamentos de la teoria de la sensación}

La respuesta de Husserl en 1900 consiste en la presentación de dos argumentos, que pueden, por otra parte, considerarse otras tantas fundamentaciones conjuntas (la segunda y la tercera) de las tesis anotadas supra bajo XII, XIII y XIV. ...

El primero se encuentra en el $\S 2$ de la Quinta Investigación. El segundo, en el $\S 7$, que fue enteramente suprimido en la segunda edición del libro.

A) ${ }^{25}$ Con independencia de la argumentación en ocho pasos que quedó antes analizada, es imposible pensar que el contenido del color objetivo y el de la "sensación" de color sea idénticamente el mismo. Cierto que el largo argumento de arriba implicaba esta conclusión también, porque, si esos contenidos fueran el mismo, ¿qué explicaría que uno sea inmanente y el otro no -es decir, que la existencia del uno sea cierta justo cuando la del otro siempre es susceptible de ser ilusoria? Pero lo que ahora nos interesa es ver que basta atender a las notas del color objetivo y a las notas de la "sensación" de color para captar la diferencia: el color objetivo es "uniforme" [gleichmässig], mientras que en la percepción misma hay un indudable "escorzarse" las "sensaciones subjetivas de color" [Abschattung der subjektiven Farbenempfindungen]. $\mathrm{Y}$ este contraste entre "uniformidad" y "escorzarse" se presenta también, invariablemente, respecto de las demás propiedades sensibles de los objetos - de las cosas en el mundo. Y la diferencia evidente en los predicados es prueba de diversidad entre los sujetos.

Naturalmente, no se puede aquí entender nada si no se toma uno mismo el trabajo de describir la situación fenomenológica a la que alude Husserl.

La uniformidad se observa, en efecto, como mentada por todos nosotros a propósito de los colores de las cosas en la vida cotidiana -en el mundo de la vida. Aunque no ignoremos las teorías físicas que han 
desterrado a los colores de la realidad, e incluso aunque las aceptemos como verdades indudables, creemos todos nosotros, por ejemplo, que nuestra piel es de un determinado matiz de color, o que nos acabamos de comprar un abrigo azul - de un azul uniforme-, o que debemos elegir el color (uniforme) de nuestro coche de entre los colores de un muestrario. En cierto sentido de la palabra 'ver', vemos constantemente cosas a nuestro alrededor dotadas de colores uniformes, o uniformemente coloreadas (de un modo tan abigarrado como se quiera).

Pero, en otro sentido de la palabra 'ver', es asimismo evidente que no vemos en absoluto esos colores uniformes que mentamos en las cosas como poseídos por ellas; que los tales colores uniformes que la vida cotidiana predica de las cosas del mundo son siempre trascendentes y jamás absolutamente dados. Son, en último término, hipotéticos. Porque lo que realmente 'vemos', en esta acepción segunda del término, son manchas de color, y ciertamente, manchas del mismo color uniforme - por pequeñas que sean-; pero "sobre" una misma cosa vemos una extraordinaria variedad de tales manchas, y muchas circunstancias modifican a la manera de un caleidoscopio el espectáculo de colores -el "campo visual". Desde ninguna posición y a ninguna luz vemos, habitualmente, un color uniforme sobre ninguna cosa. $Y$ aunque en algunas ocasiones excepcionales pueda ocurrir de otro modo, tampoco en ellas es seguro que el color dado sea el color que 'verdaderamente' -en el mundo de la vida - posee el objeto. Un cambio uniforme en la iluminación, o unas lentes harían ver un nuevo color uniforme en las cosas. $\mathrm{Y}$ no es suficiente una convención para establecer el color de los objetos —una convención del estilo de: "a la luz del día y a la distancia apropiada".

Pero la cuestión es: ¿̇en qué sentido habla Husserl de que "las sensaciones escorzan"? Lo primero que es preciso dejar establecido es que la descripción no descubre ningún otro dato que pueda denominarse "momento" de color. Hay los colores inmanentemente dados del campo visual; y los colores hipotéticos, trascendentes por necesidad, de las cosas del mundo de la vida. En todo caso - pero esto es una nueva hipótesis teórica, no un dato fenomenológico-, habrá todavía que dar algún nombre a lo que hay en la conciencia cuando "ante" ella se presenta el campo visual. ¿ $\mathrm{O}$ es que el campo visual es un trozo de la conciencia?

Pero, con toda generalidad, y dejando aparte estas últimas entidades construidas por ciertas teorías, pero siempre inaccesibles a la observación directa: ¿de qué cabe aquí decir que escorce al color trascendente y uniforme? La única respuesta, como espero que ahora se reconozca, es: los colores "inmanentes" son los únicos candidatos posibles a "escorzos" del color trascendente. Y la metáfora tiene que querer decir que los 
colores inmanentes hacen el papel de perspectivas "subjetivas" sobre el color uno y único de las cosas del mundo de la vida. Pero tan colores son los "momentos" ingredientes de color, las "sensaciones" de color, como los colores trascendentes. O, mejor dicho, las "sensaciones" de color son los únicos colores auténticamente sensibles, auténticamente dados. No es nada de extrañar que Husserl haya aquí hablado del "momento subjetivo de color, cualitativamente determinado"; porque, justamente, estas "sensaciones" son los matices individuales todos de los matices especfficos del género color, y están, por tanto, determinados cualitativamente de un modo pleno (no se puede ser directamente individuo del género: hay siempre que serlo de alguna de sus especies ínfimas).

$\mathrm{Si}$ yo supongo en las cosas del mundo de la vida colores uniformes y públicos, entonces, obligatoriamente, es porque pienso los colores inmanentes como si fueran la presentación para mí ahora y desde aquí del matiz individual realmente poseído por la cosa —el cual tiene que estar en pie de igualdad, por principio, con los matices vistos en el campo visual: lo que ocurre es que yo no puedo decidir cuál de ellos es el que realmente tiene una cosa.

Lo que aún está por explicar es qué constituye la trascendencia de los colores de las cosas. Vayamos al argumento del $\S 7$. Pero, antes, reparemos aún en el final sorprendente que tenía en manos de Husserl, en su versión de 1900, el argumento que acabamos de desarrollar: la diferencia entre Gleichmässigkeit y Abschattung "se repite respecto de todas las especies de propiedades objetivas y sus correspondientes complejos de sensación, y sólo en casos limite puede cancelarse" ${ }^{26}$ [subrayado mío].

B) En 1900, Husserl escribió estas líneas, tan escasamente compaginables con la interpretación recibida de las Investigaciones: "La doctrina de Berkeley y de Hume, que reduce a haces de 'ideas' los cuerpos que aparecen, no hace justicia al hecho de que, si bien las ideas elementales de esos haces son realizables psíquicamente, los haces mismos, los nexos de los elementos - que son los objetos de las intenciones- no han estado ni estarán jamás, en tanto que ideas complejas, presentes como ingredientes en ninguna conciencia humana. Ningún cuerpo es susceptible de percepción interna; no porque sea 'físico', sino porque, por ejemplo, la forma espacial tridimensional no es susceptible de intuición adecuada en ninguna conciencia. E intuición adecuada es lo mismo que percepción interna." 27

26 Ibid. (Hay muchos otros textos del mismo sentido en Inv. V, A.)

27 A 337. Cfr. otro texto que desapareció en 1913: Inv. II, §10 (A 128 s.). 
La premisa fundamental con la que este argumento trata de probar XII-XIV es, pues, que ningún cuerpo es susceptible de percepción interna (cuando toda vivencia sí es objeto posible de ella, o, mejor dicho, como veremos, de percepción adecuada). Y esta afirmación es en sí misma muy plausible, sobre todo cuando está tomada con la restricción "en ninguna conciencia humana". Lo que es sorprendente y discutible es el camino por el que se establece esta tesis.

Los cuerpos no son ideas complejas, porque sobrepasan en contenido, diríamos, el límite que la mente humana tiene fijado respecto de la complejidad de las ideas que pueden ocurrir en ella. No es que los cuerpos y las vivencias sean heterogéneos. Aquí no sólo no se habla primordialmente en ese sentido. Más bien al contrario, se afirma una diferencia que, aunque insalvable, sitúa a los cuerpos y a las "ideas" —o sea, a las intuiciones sensibles_ en la misma línea y aun en la misma especie. Porque las pseudoideas que son los cuerpos constan de ideas elementales que sí son "realizables psíquicamente", más un plus irrealizable psíquicamente. Es decir, ciertas propiedades de los cuerpos pueden ser partes de la conciencia referida a los cuerpos, por más que estos mismos, y, en primer lugar, algunas otras de sus propiedades, no puedan, en modo alguno, caber en la conciencia como partes de ésta (iXIV queda destruida!). Ciertamente, esto es lo mismo que decir que la distinción entre "sensaciones" y propiedades objetivas puede cancelarse; sólo que el $\S 2$ habla de "casos límite", y nuestro texto del $\S 7$ concede tanto al fenomenismo clásico que parece que los casos excepcionales se ven ahora tratados como la regla.

De nuevo, los únicos candidatos a "idea elemental realizable psíquicamente" son los colores, las formas y los tamaños del campo visual, más los sonidos en tanto que datos sensibles estrictos, más... el resto, en definitiva, de las "ideas de la sensación" clasificadas en el Ensayo por Locke. Por cierto que la "forma espacial tridimensional", a pesar de lo que dice Husserl, debería figurar en esta lista, porque no es menos absurdo decir de la conciencia que es roja o caliente o lisa, que decir que tiene tres dimensiones espaciales; y, en cambio, tan adecuadamente (tan inmanentemente) está dada la "forma espacial tridimensional", como adecuada (inmanentemente) están dados los demás miembros de este conjunto. Lo que de un cuerpo no puede darse adecuadamente está mal descrito aquí.

Es evidente que las conclusiones chocantes a las que Husserl se ve conducido no son de ninguna manera gratuitas. Husserl sostenía en 1900 que los cuerpos existen. Pero sería absurdo afirmar la existencia de algo que no pueda ser conocido ni aun por un sujeto cognoscente absolutamente perfecto. Si empleamos para él la palabra 'Dios', como tantas 
veces ha hecho la filosofía moderna, si los cuerpos existen, deben poder ser conocidos adecuadamente por Dios siquiera. $\mathrm{Y}$ es evidente - perdónesenos de nuevo que repitamos lo que piensa Husserl sin intentar desde un principio fundamentarlo o refutarlo- que el hombre - también existen hombres - no es capaz de obtener conocimiento adecuado de cuerpo alguno, ni siquiera del propio por dentro.

Pero en este punto entra en acción cuanto expusimos antes: si es evidente y cierto que mi conciencia humana vive referencias intencionales a cuerpos. Hay percepción adecuada de la percepción de cuerpos, la cual, a su vez, es inadecuada. Y parte de lo captado adecuadamente es que creo percibir tal cuerpo de tal color - que se me da en escorzos variadísimos de colores en el campo visual-, tal figura -escorzada también, y de la misma manera, y siempre trascendente a todo escorzo suyo que yo capto adecuadamente-, tales y tales propiedades táctiles - cuyos escorzos son lo único que poseo inmanentemente-, etc.

Si no puedo echar mano más que de dos nociones (acto ingrediente en la conciencia y objeto trascendente), debo concluir que todo lo que se da a la percepción adecuada es parte de la conciencia (intencional o no). $Y$ en el acto mismo tendré que distinguir algo inmanente que haga posible la referencia a lo trascendente ("aprehensión objetivante") y otra parte, para la que se halla dispuesto el término clásico "sensación" (por más que deba afrontar la consecuencia de declarar rojas, frías o aromáticas a las sensaciones; al menos tengo los ilustres precedentes de los empiristas clásicos, que incluso llegaron mucho más allá en este camino).

Por otra parte, en auxilio de Husserl bien podían venir algunas otras consideraciones. Ante todo, la idea de que la captación adecuada de lo que él llamaba sensaciones sólo podría, por principio, ser posible una vez que se hiciera abstracción de toda aprehensión objetivante. Los colores inmanentes, por ejemplo, sólo escorzan el color transcendente cuando la conciencia, en vez de limitarse a tenerlos o vivirlos, se dirige mediante ellos hacia objetos transcendentes. Las sensaciones resultan entonces, por así decir, proyectadas hacia la trascendencia. Sin venir a formar parte de ella, son, como escribió Husserl en otro lugar de las Investigaciones, materiales análogos de construcción ${ }^{28}$ con los que se "fabrican" las propiedades trascendentes de las cosas a las que damos aún el nombre de "propiedades sensibles". Son antes, en cierto sentido, los escorzos que las sensaciones; o sea, los colores empiezan por ser los colores de las cosas, hasta que las ilusiones, las variaciones caprichosas, etc., nos enseñan a captarlos como puras "sensaciones" husserlianas, libres de toda función en la construcción consciente del mundo externo.

28 Inv. I, $\$ 23$ (A 75). 
Pero que se produzca cierto desconcierto cuando a uno le piden que observe las sensaciones desprovistas de toda aprehensión objetivante no es obstáculo para que el análisis determine en qué consisten todas estas partes inmanentes (del modo como hemos mostrado en las discusiones precedentes).

Otro problema muy distinto es si realmente la percepción de las "sensaciones" no exige algo más que el puro vivirlas; es decir, si no exige algo que denominaríamos "aprehensión objetivante" a no ser porque Husserl ha reservado este término a la "interpretación" de las "sensaciones" capaz de levantar ante la conciencia un objeto trascendente. $\mathrm{Y}$ en la percepción adecuada parece resueltamente absurdo hablar de nuevo de un "fenómeno" compuesto de sensaciones y aprehensión de ellas en tanto que presentaciones de cierto objeto.

La cuestión siguiente es, justo, el análisis de la percepción adecuada, que aún es obligatorio si queremos probar que nuestra interpretación es la correcta. Pero tengo todavía que dejar constancia de dos problemas laterales que han aparecido de hecho en los textos citados.

El primero está en la llamativa vacilación del $\S 7$ de Husserl. Primero se dice que la conciencia humana es incapaz de realizar las ideas en que tendrían que consistir los cuerpos, según el fenomenismo. $\mathrm{Y}$ en la frase siguiente se afirma que la imposibilidad de la intuición adecuada - que estamos suponiendo equivalente a realización psíquica, o, mejor, concomitante necesario de ésta_ es absoluta, no restringida al género humano o a la subjetividad finita.

Creo que la modificación de punto de vista tiene su razón evidente en que el realismo ingenuo que Husserl sostenía en 1900 es incompatible, como antes dije, con la intrínseca imposibilidad de que los cuerpos sean adecuadamente perceptibles. Pero puesto que percepción adecuada vale tanto como "realización psíquica", si los cuerpos son intuibles adecuadamente, entonces habrá que decir que hay al menos una conciencia que los tiene como partes suyas (que los percibe internamente, como dice el texto). La propiedad que impide que la conciencia humana alcance este conocimiento será, en tal caso, sensorium Dei, cuando no a.tributo o modo del cognoscente perfecto.

No es extraño que Husserl, tan poco amigo - sobre todo en torno a 1900 - de las especulaciones metafísicas, retrocediera ante semejante consecuencia. Pero el realismo, unido a su teoría de la percepción adecuada, le llevaba con todo rigor a ella. En 1913, la salida de la dificultad es el idealismo. Pero habrá que comprobar hasta qué punto el dilema había cambiado en ese año. Es decir, hasta qué punto la teoría de la evidencia sostenida por las Investigaciones lógicas había sido abandonada.

El segundo interesantísimo problema lateral aparece cuando se exami- 
na con cuidado la lista de las características que Husserl predica abiertamente de sus "sensaciones" - y que no incluye lo que el análisis obliga a decir también (o en vez de). Las propiedades reconocidas a toda sensación son: a) ser parte abstracta de las percepciones externas o inadecuadas -dejando a un lado el hecho de que también pueden hallarse las sensaciones en la conciencia en misiones distintas de ésta y menos importantes-; b) ser reell. Este adjetivo alemán es de difícil traducción; y especialmente en el capítulo de las Investigaciones que nos ocupa, ya que en el no había aún alcanzado sentido unívoco ni verdadero valor terminológico. Aquí quiere decir "realmente presente en" un todo cualquiera, o de la conciencia o del mundo. Pero la tendencia clara es a reservarlo como equivalente de "vivido", "consciente", "fenomenológico", frente a real, que se aplica a lo realmente constitutivo de un todo mundanal extraconsciente. ${ }^{29} \mathrm{He}$ decidido seguir a José Gaos en el uso de 'ingrediente' para traducir reell; pero debe entenderse que, siempre que empleo esta expresión con carácter terminológico, me refiero a ingrediente en la conciencia', a parte de la conciencia.

En cualquier caso, lo que es reell es temporal. Pero lo que es parte abstracta es individuo de una especie, como se prueba en la Investigación Tercera. ${ }^{30}$ Pero lo que es individuo de una especie no puede estar hecho únicamente de tiempo, ya que estar bajo una especie - ser abstracto o no-independiente- es estar sometido al menos a una ley necesaria, y el puro tiempo es la pura contingencia, la pura no-necesidad. Será imprescindible tratar de todo esto mucho más minuciosamente; ${ }^{31}$ pero lo que importa desde ahora es tener presente que la descripción fenomenológica suscita, incluso en los niveles que menos propicios a esto parecen ser a la distancia, el problema ontológico cuyas dos caras son la "participación" (= la naturaleza de lo universal) y el principio de la individuación (= la naturaleza de lo individual).

\section{§4. La percepción adecuada}

En la expresión 'percepción adecuada', adecuación significa realización plena de la aspiración contenida en la naturaleza de todas las percepciones, cumplimiento total de la intención perceptiva. $\mathrm{Y}$ "toda percepción se caracteriza por la intención de captar su objeto como presente él mismo, exactamente tal como es". ${ }^{32}$

Lo que interesa es considerar los criterios que Husserl propone para

29 Cfr. Inv. V, §16 -en especial, la nota al final- (A 375).

30 Cfr., sobre todo $\$ \$ 7$ y $10-12$.

31 Cfr., la nota 16.

32 Inv. V, §5 (A 333). 
la adecuación -y que, en lo que tienen de más original, están diluidos en la redacción de 1913.

El primero de ellos no ofrece por sí solo mayor problema. Ya que consiste, sencillamente, en no introducir en el objeto, mediante la aprehensión, nada que no esté "representado ello mismo intuitivamente" $3 s$ en el acto perceptivo. En otras palabras, una intuición es adecuada cuando no contiene más mención vacía o simbólica que dato auténtico; cuando no cree que su objeto posea partes o propiedades que no están dadas de hecho simultáneamente.

En cambio, el segundo criterio no sólo es sorprendente, sino del todo impracticable - aunque perfectamente concorde con cuanto llevamos visto en los análisis precedentes. Pues Husserl exige también para la adecuación que el objeto esté "dado ingredientemente" en la percepción [reell gegeben $]^{34}$ Es decir, que sea $o$ bien todo o bien una parte del acto perceptivo mismo referido a él.

Que éste sea el significado de 'reell gegeben', y que no suceda que me dejo llevar demasiado lejos por mi interpretación de un adjetivo quizá ambiguo, es cosa que manifiestan plenamente los sinónimos de Husserl para esta expresión. Uno de ellos es también neutro: 'leibhaftig gegenwärtig', o sea, "presente en persona". Pero el segundo es: 'im Wahrnehmen selbst gegenwärtig und mit ihm eins', es decir: "presente él mismo en el acto de percepción y uno con él".

Pero con más claridad aún que esta frase, el pensamiento se expone en la línea siguiente: "va pues, de suyo, es evidente a partir del mero concepto de percepción, que sólo la percepción interna puede ser percepción adecuada; que sólo ella puede dirigirse a vivencias dadas a la vez que ella y pertenecientes con ella a una misma conciencia". ${ }^{35}$

La segunda condición quizá pueda estar cumplida por cualquier acto - e incluso por cualquier vivencia en general- simultáneo de otro. $\mathbf{Y}$ es seguro que la satisfacen todos los todos vivenciales respecto de cada una de sus partes. Como, sin embargo, es absurdo decir que todas las vivencias concretas que poseen partes son percepciones adecuadas, es necesario requerir a la vez la primera condición. Es decir, que el acto aprehensor no sólo sea simultáneo con la vivencia objeto suyo, sino que se refiera intencionalmente a ella, y esto, no simbólicà, sino intuitivamente.

La primera de las condiciones de la adecuación, por tanto, extiende, como no podía ser menos, a la percepción adecuada la estructura inten-

33 Ibid.

34 Ibid.

35 Ibid. La noción fundamental de Fülle confirma plenamente esta interpretación. Pero es necesario dejar la prueba de este punto a la continuación del presente estudio. 
cional básica propia de todos los actos - $\mathrm{y}$, expresamente, de la percepción inadecuada. Pero, a un tiempo, la segunda condición nos informa de que la intencionalidad adquiere su perfección, o llega a su cumplimiento, al menos en uno de los órdenes más importantes que cabe considerar a propósito de ella, cuando el objeto intencional es una parte ingrediente del acto intencional mismo (o es idéntico con éste como un todo). Justo en tanto que el objeto permanece trascendente al acto -no inmanente en él como ingrediente suyo-, la percepción, se nos dice, no realiza lo que por su esencia misma pretende. $Y$ ello no es sino lo que hemos oído en los pasados argumentos, una vez con la presentación del caso límite, y otra vez más bien como si la regla fuera la realización psíquica de algún repertorio de propiedades del objeto que, como tal, permanece trascendente.

Por otro lado, si no distinguimos más que contenido ingrediente o fenomenológico o vivido, y objeto o contenido intencional, es imposible que nuestra conclusión difiera de la de Husserl. Todo lo más, podríamos negar que haya percepciones adecuadas; pero aún así deberíamos conceder, irremediablemente, que el mero concepto de tal cosa es el que Husserl acaba de exponer. Por cierto, aunque esto haya de ser dicho ahora puramente entre paréntesis, es una cuestión de extraordinaria importancia examinar lo que la fenomenología posterior a las Investigacia nes es capaz de afirmar sobre este mismo punto, verdadera piedra de toque del valor de cualquier teoría del conocimiento.

La teoría intencional de la percepción adecuada y la teoría de ella en términos de todo y parte son estrictamente incompatibles. Ante todo, hay que descartar por absurda, en cualquier sentido en que se la tome, la idea de que acto y objeto pudieran coincidir absolutamente. No habría entonces otra cosa en la conciencia que percepciones adecuadas; pero lo serian de absolutamente nada. (Desde luego, la posición de Brentano - la distinción de dos "direcciones de la percepción"- no está gravada por esta clase de imposibilidad.)

Sólo cabe, en un principio, examinar si de alguna manera el objeto inmanentemente dado puede ser una parte del acto referido a él. El carácter perceptivo queda eliminado, desde luego. Resta el "fenómeno". Pero la "aprehensión" es imposible que coincida con su objeto, pues precisamente son distintos los objetos de la percepción adecuada y del acto adecuadamente captado - si es que es un acto. Y la diferencia de objetos sólo puede deberse a la diversidad del "como qué" (inmanentemente dado) se los mienta, o sea, a la diversidad de la aprehensión.

- Nos vemos remitidos, como nos sucedió ya en realidad en los parágrafos de arriba, a la "sensación" o "contenido primario" como único candidato a tener en cuenta. Una percepción adecuada, si lo es cuando 
su objeto es "uno con ella", cuando está "ingredientemente dado", tendrá que serlo gracias a que el objeto se realice en ella al modo de la parte real de ella que funda la aprehensión, la intención. Pero ello no es menos absurdo que las hipótesis antes examinadas. Es ininteligible que la materia que viene a informar la aprehensión sea justamente el objeto intencional que se ofrece frente al acto gracias a la aprehensión. Y si pudiera ocurrir que contenido primario + aprehensión fuera igual a "fenómeno adecuado del contenido primario", sucedería: 1) que "fenómeno adecuado del contenido primario' no es de ningún modo lo mismo que 'contenido primario'; y 2) que jamás podríamos saber por medios descriptivos (= fenomenológicos) que las cosas hubieran ocurrido de esa manera precisamente. Porque nunca tendríamos acceso directo al contenido primario mismo, y, asi, nunca podríamos enterarnos realmente de que el resultado intencional está adecuadamente designado con el nombre 'fenómeno adecuado del contenido primario'. Tendríamos una teoría, que sólo sería plausible si pudiéramos mostrar que es condición evidente de la posibilidad de algún dato evidente en sí mismo de modo adecuado. Por cierto que ésta es la vía por la que, de avanzar más, llegaría a mostrarse en seguida el carácter no fenomenológico de muchas de las tesis sostenidas por Husserl en 1900 en las grandes Investigaciones dedicadas a la fenomenología. Dejemos este asunto capital para cuando los análisis hayan progresado suficientemente.

\section{§5. Yo fenomenológico y tiempo}

Es bien conocido que el primer capítulo de la Investigación Quinta contiene dos polémicas: el rechazo de la teoría neokantiana acerca del yo puro -en especial, en la forma que recibe en la psicología de Paul Natorp-; ${ }^{36}$ y el abandono de varios puntos cruciales en la psicología descriptiva de Brentano: la negativa a reconocer que todo fenómeno psíquico tenga carácter intencional y sea objeto de la conciencia interna.

Husserl acusa a Brentano, respecto de la última cuestión, de haber dejado a un lado la pura descripción, para conceder el primer puesto a la teoría. Él, por su parte, no ve motivo alguno por el cual desechar de antemano la posibilidad de vivencias totalmente desatendidas. ${ }^{37}$

La no intencionalidad de muchos fenómenos psíquicos, o, mejor (en vista de que no todos ellos son necesariamente verdaderos fenómenos ante la conciencia interna), de muchas vivencias, debe ser examinada en detalle. Pero hay que recordar que ha sido asumida por nosotros desde el momento en que hemos aceptado que en un acto se encuentran

36 Vid. sobre todo $\S 8$.

37 Inv. V, §5 (A 334). Cfr. en general Inv. V, § 10. 
partes, todas ellas vividas también; siendo asi que sólo el acto como un todo, y justo gracias a alguna de sus partes, es una vivencia intencional.

Natorp aducía dos fundamentos para la defensa del yo puro. En primer lugar, el dato invariable, que acompaña a todo contenido de conciencia, que consiste, justamente, en su ser contenido de la conciencia propia, en pertenecer a la unidad de mi conciencia. A este elemento que comparten todas las vivencias de una misma conciencia, lo llama Natorp Bewußtheit (calidad de consciente). Y afirma que es la relación que todas las partes del yo empírico o conciencia tienen respecto del sujeto puro -incapaz de tornarse objeto en ningún sentido- que es el yo puro. El cual, por otra parte, como sujeto trascendental, desempeña la misión de condición de posibilidad de la unidad de la conciencia. La multiplicidad inmensa de los contenidos de la conciencia necesita de un principio unificador, libre él mismo tanto de la multiplicidad como de la posibilidad de ser un contenido más entre el resto. Como Kant explica en la Critica de la razón pura, las síntesis empíricas todas tienen el fundamento de su posibilidad en una síntesis suprema y no empírica, que es, justamente, la síntesis de la apercepción trascendental, "el yo pienso que tiene que poder acompañar a todas mis representaciones". 38

El argumento en contra más interesante que Husserl expone es, sin duda, que la conciencia, el yo fenomenológico, como él dice, no precisa de ninguna sintesis trascendental que funde su unidad. La conciencia es un continuo que 'se unifica por sí solo. "Va de suyo que el yo no es nada que posea una índole peculiar y que se cierna sobre la multiplicidad de las vivencias; sino que, simplemente, es idéntico con la unidad por vinculación de todas ellas." 30

Una unidad por vinculación (Verknüpfungseinheit) está definida ontológico-formalmente como un todo cuyas partes de primer orden son trozos, que fundan entre todos un "momento" responsable de la unidad del todo. ${ }^{40} \mathrm{Y}$ Husserl describe, en el $\S 6$, precisamente en estos términos el todo que es el yo fenomenológico.

Las "unidades coexistentes", los presentes de la conciencia, aparecen aquí comó los trozos del yo. Cada uno de estos presentes, a su vez, puede consistir o bien en un todo cuyas partes de primer orden sean trozos, o bien en un todo cuyas partes de primer orden sean "momentos". Lo que no se nos dice -y posiblemente no se nos pueda decir- es de qué tipo cabe pensar que sea el "momento" unificador de los trozos en una "unidad de la coexistencia". Pues si la respuesta fuera: el ahora, enton-

$38 \mathrm{KrV}$ B 131-140.

39 Inv. $\mathrm{V}, \S 4$ (A 331).

40 Inv. III, $\$ 21$ (A 269). 
ces ignorariamos cómo una unidad formada por puros "momentos" puede llenar también ella un ahora. Quizá, dada la naturaleza de las "sensaciones" que nos han puesto de manifiesto los parágrafos anteriores, Husserl pensaba aquí en cualquier "momento figural" (Gestaltqualität) -incluidos, sobre todo, los no temporales. ${ }^{41}$ Sería, desde luego, interesantísimo probar que era así.

Pero, por el momento, nuestra tarea es analizar qué parte no-independiente fenomenológica unifica por vinculación el yo y, como ocurre en el empirismo humeano, expulsa de la filosofía al yo puro de los seguidores de Kant. Husserl presenta su respuesta en el texto sin duda más difícil de todo el capítulo primero de la Investigación Quinta: "Las unidades de coexistencia desembocan constantemente las unas en las otras de punto del tiempo en punto del tiempo. Constituyen una unidad de variación, que exige, por su parte, la permanencia constante o la modificación constante de al menos un 'momento' esencial para la unidad del todo $\mathrm{y}$, por consiguiente, inseparable de él en tanto que todo. Este papel es desempeñado primordialmente también por la conciencia subjetiva del tiempo, entendida como escorzo de las 'sensaciones de tiempo'; la cual, por paradójico que paręzca, presenta una forma omnicomprensiva del instante de la conciencia, o sea, una forma de las vivencias que coexisten en un punto objetivo del tiempo." 42

La conciencia subjetiva del tiempo es, pues, el "momento" que vincula los trozos (coexistencias) del yo. Se dice de ella que "también" desempeña este papel, es de suponer que aludiendo al que juegue en la unificación de las unidades mismas de la coexistencia - por difícil que sea precisarlo. Pero lo sorprendente es que no se diga que el vínculo es el tiempo, sino "la conciencia subjetiva del tiempo". Ella es la que hace de forma omniabarcante del instante consciente. $O$, si darstellt debe ser entendido en otra acepción, ella es la que constituye, merced a las "sensaciones de tiempo", la forma en cuestión. El texto creo que impone la primera manera de interpretar el verbo alemán; pero, en mi opinión, lo que realmente se quiere decir no difiere apenas de lo que uno lee si acepta la segunda posibilidad.

En efecto, se nos indica también que la conciencia subjetiva del tiempo, en esta función capital, debe ser comprendida como Abschattung der "Zeitempfindungen". A pesar de que la traducción castellana literal que acabo de insertar oculte el asunto del que se está realmente hablando, el análisis sobre los escorzos sensibles que ya hemos realizado nos pone inmediatamente en la pista de la idea de Husserl. La conciencia subjetiva del tiempo es comparada aquí a una percepción ex-

11 Cfr. Inv. III, $\$ 22$.

42 Inv. $V, \S 6$ (A 396). 
terna. La impropiedad de la comparación, o, más bien, la analogía de los términos comparados, se marca usando comillas para las "sensaciones de tiempo". Éstas funcionan en la conciencia subjetiva del tiempo de alguna manera análoga a como las sensaciones - recuérdese nuestra interpretación- lo hacen en la percepción inadecuada. Sólo que la conciencia del tiempo no está, por lo visto, compuesta por otras partes que no sean "sensaciones de tiempo". ¿Qué son éstas? Y, sobre todo: ¿qué escorzan?

Las "sensaciones de tiempo" son el escorzo en la inmanencia de una propiedad trascendente que debe hallarse respecto de ellas en la misma razón en que el color trascendente se halla respecto de los colores inmanentes. Luego la respuesta que buscamos tiene que ser: las "sensaciones de tiempo", la conciencia subjetiva del tiempo, es el tiempo inmanentemente dado; y lo escorzado por ellas es también tiempo, pero tiempo trascendente. No el tiempo ingrediente en el que está el yo fenomenológico; sino el tiempo trascendente que, sin que se dé adecuadamente, es predicado por el sujeto de los (o de ciertos) objetos trascendentes.

El tiempo dado absolutamente es, a la vez, flujo y ahora continuo. La forma en que siempre se nos dan originariamente todas nuestras vivencias es la forma plenamente inmutable del ahora. Jamás hemos vivido en otro tiempo que ahora. El ahora no cesa, y representa, como dice el texto, una forma que abarca todos los "instantes" conscientes, o, lo que es lo mismo, una forma que es la condición de la posibilidad de toda coexistencia - y, a fortiori, de todo fluir de los instantes los unos en los otros. Pero el tiempo en tanto que dato adecuado no es sólo ahora inmutable. Precisamente, si se redujera a esto, no sería tiempo, porque no se daría en él sucesión continua. Muy al contrario, la experiencia absoluta de la inmutabilidad del ahora es, exactamente, conciencia de que, mientras pasa el tiempo, el fluir de un instante en otro permanece sin modificación. Es imposible captar la quietud del ahora, si no se aprehende, juntamente con ella, el fluir de lo que llena el ahora (el fluir de los "ahoras", si se permite la expresión). Lo que significa que el presente inmanentemente dado es siempre más que puro instante, porque retiene ahora un poquito del pasado y proyecta ahora otro poquito del futuro. El paso del tiempo a través del ahora fijo es lo que, en total, constituye el verdadero dato absoluto.

Una vez que una materia consciente cualquiera llena la forma del tiempo -el fluir a través del ahora inmóvil_, pasa en adelante a ocupar un puesto determinado en la serie del pasado. La cual va, toda ella, hundiéndose, por así decirlo, cada vez más, como la costa de la que el barco se aleja; pero constituye una totalidad inmodificable. $O$, mejor 
dicho, una totalidad cuya única modificación continua es su escorzarse temporal.

Sin embargo, el tiempo trascendente que se escorza en el tiempo inmanente (o, lo que es lo mismo, en la conciencia subjetiva del tiempo) no puede ser la serie intangible del pasado, en la medida en que sea este pasado consciente como tal. Esa solidez, esa especialización sufrida por el pasado no alcanza - a no ser que se trate de puro pasado reconstruido hipotéticamente, pero no dado- a que haya que declarar tiempo trascendente a todo el pasado. El tiempo no fenomenológico no es otro que el que permite hablar de la simultaneidad entre entidades que no son vivencias coexistentes del mismo yo fenomenológico. Las "unidades de coexistencia" que se establecen tanto en el mundo de la vida como en el mundo de la ciencia son los "instantes" fluyentes de un tiempo supuesto, pero siempre trascendente al dato temporal adecuado y absoluto.

El primer objeto trascendente soy yo mismo en cuanto persona que forma parte del mundo.

\section{§6. Realismo de la actitud natural}

El modo como la reducción fenomenológica es introducida en los escritos posteriores de Husserl ${ }^{43}$ se hace sólo propiamente comprensible cuando se tiene en cuenta el marco general metafísico que el filósofo defendía en torno a 1900. El capítulo primero de la Investigación Quinta es seguramente el lugar en que fue expuesto con más claridad.

Todos los seres reales pertenecen al mundo. El mundo en sí [die Welt an sich] es el correlato objetivo de la ciencia, pensada en su conclusión perfecta [die Welt der ideal vollendeten Wissenschaft].

El mundo es, por otra parte, el conjunto total de los objetos empíricos o fenoménicos [phänomenale]. Y cada uno de estos miembros del mundo es una cosa [Ding]. Al mundo mismo se le aplica en alguna ocasión el adjetivo "fenoménico".

Las cosas que componen el mundo real son de dos clases: cosas psíquicas o personas, y cosas físicas o cuerpos [Personen, Körper]. Entre las cosas psíquicas del mundo me encuentro yo mismo: el yo fenoménico o empírico [empirisches, phänomenales Ich]. En este sentido de la palabra 'yo', yo estoy compuesto de dos partes: mi cuerpo orgánico y vivo [Ichleib] y el yo puramente psíquico, el yo espiritual, el alma [seelisches Ich], el individuo psíquico. ${ }^{44} \mathrm{El}$ yo fenomenológico, la conciencia es, precisa-

43 Interesa ante todo Die Idee der Phänomenologie. Vid. Hua. II (ed. W. Biemel) (Den Haag 1950).

44 La serie de breves citas alemanas en éste y los dos párrafos anteriores corresponde a: A 337 (los dos primeros textos), A 328 (los tres siguientes), A 331 y A 342. 
mente, "el acervo total fenomenológico del yo espiritual". ${ }^{45}$ Por tanto, una parte del "alma", que se obtiene dejando en ésta de lado cuanto no es dato puro en el tiempo subjetivo. Y, como parte del "alma", el yo fenomenológico es parte del yo fenoménico, y, a fin de cuentas, parte del mundo.

Las secciones del $\S 4$ suprimidas en $1913^{46}$ son una introducción al problema esencial de las relaciones concebibles entre el yo fenomenológico y el empírico. Distingue en ellas Husserl: el yo fenomenológico del instante, el yo fenomenológico en el tiempo extendido y el yo como objeto permanente [verharrender Gegenstand]. Este último término es sinónimo de "alma".

El yo fenomenológico del instante es el cogito actual adecuadamente aprehendido. El yo fenomenológico en el tiempo extendido es el correlato de la "evidencia o de la probabilidad evidente 'yo fui' ". El yo permanente, en cambio, es una unidad "ya no fenomenológica, sino que se. halla en el ámbito de las leyes causales" [sie liegt in kausaler Gesetzlichkeit]. Precisamente es ésta la propiedad fundamental de cuanto es una cosa [Ding]. Los miembros del mundo están todos en un sistema de conexiones temporales y causales, que es, justo, lo que da lugar a "la unidad de una cosa en sentido metafísico" [eine dingliche Einheit im metaphysischen Sinne]. "Desde luego debemos dejar abierta la cuestión de si las cosas psíquicas y las físicas pueden distinguirse $-\mathrm{y}$ ello, de qué modoen forma tal que queden unas junto a otras, en pie de igualdad, como unidades cósicas. Aquí sólo nos interesa lo fenomenológico, y es seguro que el yo fenomenológicamente reducido... porta en sí mismo su unidad, valga o no, en la consideración causal, como una cosa."

La permanencia del alma a través de la modificación del yo fenomenológico de instante en instante no debe ser confundida con la permanencia del ahora fenomenológico mismo - descrita en nuestro parágrafo anterior-. Creo que los datos impiden aceptar la posibilidad, que tienta en un principio, de sostener que el ahora no fluyente es justo el ahora del alma, que se escorza en el puro flujo en que consiste el tiempo fenomenológico. Por más que el ahora esté detenido y el paso por él fije, espacialice en cierto sentido, los contenidos conscientes, la pasada descripción permite superar estas apariencias. El yo psíquico permanente trata de responder a necesidades teóricas planteadas por otros datos, y, en especial, por el realismo metafísico. (Sería mera especulación aducir los hábitos y las facultades como otros motivos: Husserl no dice nada al respecto).

Ahora bien, en los $\$ \S 6$ y 8 encontramos nuevos textos plenamente 45 Inv. V, § 1 (A 325).

46 A 332. Las citas que siguen son de este texto. 
explícitos, sobre la relación entre el yo fenomenológico y el yo empírico. No importa, últimamente, que el yo fenomenológico admita o no una consideración causal que permita hablar también de él como una cosa, en el sentido metafísico de unidad temporal y nudo del sistema universal en la causalidad. El marco metafísico que supone Husserl obliga a decir lo que escribi líneas arriba. Y, en efecto, leemos: "En el juicio 'yo soy', la evidencia afecta a cierto núcleo, no delimitado conceptualmente de modo riguroso, de la representación empírica del yo." 47 Es decir: el yo fenomenológico del instante es un sector —el adecuadamente dado-del yo fenoménico. A lo que debe objetarse que tal afirmación es un paso en la metafísica incomparablemente más gratuito que el que Descartes dio al suponer que la cogitatio actual es un modo de la sustancia pensante.

Aún ha expresado Husserl la misma idea - no acompañada nunca de un intento de fundamentación - con más claridad en el parágrafo dedicado a la polémica con Natorp. El yo fenomenológico "se comporta respecto del yo anímico exactamente como lo hace "el lado que entra en la percepción' de una cosa externa percibida, respecto de la cosa entera". ${ }^{48}$ "La autopercepción del yo empírico es algo cotidiano, que no presenta problema alguno de comprensión. El yo se percibe lo mismo que se percibe cualquier cosa externa. Que el objeto no caiga dentro de la percepción con todas sus partes y todos sus aspectos, no es óbice alguno para ello, ni en un caso, ni en el otro." 49 Y, a propósito de esto último, Husserl pone el ejemplo del propio percibir adecuado, que no cae él mismo dentro de lo que se percibe adecuadamente, cuando, sin embargo, pertenece al yo -incluso pertenece a su contenido fenomenológico-. Sería inútil añadir más textos, ${ }^{50}$ en los que abunda el capítulo entero (en especial, véase el comienzo del $\$ 7$, en el que se encuentra el punto de partida de la posición que defendió treinta años más tarde Jean-Paul Sartre en su ensayo De la Transcendance de l'Égo).51

La fenomenología, en torno a 1900 , no era más que la parte primera de la psicología, y, a la vez, el fundamento de la división entre psicología y ciencia de la naturaleza, y el fundamento de la teoría del conocimiento. Es perfectamente consecuente, desde este punto de vista, rechazar la identificación de psicología y ciencia de los fenómenos psíquicos, porque la psicología se refiere a un dominio de objetos que desborda con mucho los límites de lo fenomenológico; de modo semejante, re-

47 A $334 s$.

48 A 342.

49 A 343.

50 Cfr., aún, sin embargo, Inv. V, $\S 12$ (A 355 s.).

51 Vid., ahora la edición de S. Le Bon (París, 1972). 
cuerda Husserl, a como la ciencia de la naturaleza no tiene el derecho pleno de llamarse ciencia de los fenómenos físicos, pues si bien comienza por ellos, se extiende luego a muchos más objetos, cuya realidad está reclamada (por ejemplo, causalmente) por la realidad de los puros fenómenos.

\section{§7. La clasificación fundamental de las ciencias}

Las vivencias intencionales constituyen, pues, "el núcleo fenomenológico esencial del 'yo' fenoménico". ${ }^{52} \mathrm{Y}$, en consecuencia, todas las ciencias que estudian el ser real se hallan en continuidad fundamental. En ellas no se encuentra diferencia mayor que la que separa los análisis descriptivos puros, de las teorías que dan cabida a consideraciones causales y a entidades que no se ofrecen intuitivamente. ${ }^{63}$ Pero no sería justo situar una fenomenología física en coordinación estricta con una fenomenología psíquica, ambas en pie de igualdad. Al contrario, la diferencia básica entre todos los fenómenos temporales es la trazada, inmutablemente, entre los susceptibles de intuición adecuada y los que no la consienten por principio. Lo que viene a ser lo mismo que distinguir entre fenómenos auténticos y pseudofenómenos. Los fenómenos real y exhaustivamente fenómenos son los llamados fenómenos psíquicos (intencionales y no intencionales). Por consiguiente, la única fenomenologia digna de este título es la que los estudia a ellos en los límites exactos de su intuición adecuada; ${ }^{54}$ es decir, con un método puramente descriptivo, abstracción hecha de consideraciones genéticas (causales). $\mathrm{Y}$ ello, con tanto más fruto científico, cuantas más conexiones necesarias o de esencia pueden ser aprehendidas sobre la base de los fenómenos adecuados. Porque -y de aquí la extraordinaria importancia de la obra de Brentano en el origen de la filosofía del siglo XX - hay verdaderamente leyes que no son leyes causales, sino regularidades, estrictamente necesarias, que afectan a la pura naturaleza de las partes de los fenómenos. La fenomenología puede ir mucho más allá del simple registro de sucesos individuales y contingentes, justo porque todo ser individual y contingente tiene su eidos propio, sin cuyo conocimiento carece de sentido cualquier cuestión genética. Si no fueran los fenómenos de la conciencia los únicos que pueden darse adecuadamente, la fenomenología, tal como era concebida por Husserl en la huella de Brentano, se extendería sin dificultad metódica ninguna a otros campos que los que de hecho puede abarcar.

Junto a la lógica pura (pensada en su máxima amplitud, desde la gra-

52 Inv. $V, \S 8$ (A 348).

53 Cfr. Inv. V, $\$ 7$ (A 338 s.).

54 Cfr. Inv. V, $\$ 17$ (A 376). 
mática lógico-pura al estrato superior de la mathesis universalis) ${ }^{85}$ y las ciencias aprióricas que estudian leyes sintéticas de no-independencia de abstractos de diversas indoles, ${ }^{56}$ las ciencias de los seres reales presentan, pues, una estructura muy simple. En la base, la fenomenologia. Sobre ella, la psicología, en una dirección, y la ciencia de la naturaleza, en la otra (ambas dedicadas al estudio de "cosas"; es decir, abarcando por principio la consideración causal de sus objetos). La fenomenologia, obligada por el ideal de la adecuación a reducir la consideración cósica de la conciencia, trata de entes reales en una notable restricción. Sus objetos están en el tiempo, pero son aprehendidos como si no tuvieran que ver con el entramado causal que define al ser real en sentido pleno.

En cuanto a la relación entre el ser ideal (analítico o sintético, tanto da en este caso) y el ser real, siempre es la misma: el ser ideal es especie respecto del ser real, que es individuo. $Y$, como los individuos adecuadamente dados son las vivencias propias, las ciencias aprióricas que tienen preeminencia gnoseológica son, justo, todas aquellas cuyos individuos son vivencias (objetos no sólo reales, sino, además, "ingredientes"). Lo que sucede es que en esta situación se hallan todas las ciencias aprióricas de mayor interés y más larga historia: la lógica (= mathesis universalis), la ética, la estética (es decir, las disciplinas teóricas y las axiologías que están en la base de las ciencias normativas y prácticas fundamentales). ${ }^{67}$

Será el examen de la noción de esencia defendida por Husserl en 1900 el que aporte las últimas justificaciones imprescindibles.

\section{§8. Tiempo trascendente y tiempo inmanente}

Este cuadro general de la metafísica y de las ciencias pone muy claramente de manifiesto cuáles eran las articulaciones más débiles del pensamiento de Husserl en la época en que fueron escritas las Investigaciones lógicas. Especialmente, hace claridad, como dije antes, sobre el modo en que era forzoso que irrumpiera la idea de la reducción fenomenológica.

Hemos visto aparecer en los textos citados el término 'reducción', aplicado, precisamente, a la obtención del yo fenomenológico partiendo del yo-cosa o alma. Pero hemos hecho hincapié en el hecho de que esa reducción es, sobre todo, una abstracción de la consideración genética. El residuo de semejante reducción pertenece a la misma cosa real en la que se practica la reducción. Leamos aún este fragmento: "También las vivencias psíquicas y los yoes se acreditan, por lo que hace a su ser y a sus conexiones según leyes, únicamente en la ciencia en tanto que sistema

55 Cfr. Prol. $\$ \$ 67.69$.

so Cfr. Inv. III, $\$ \$ 7$ y 10-12; Inv. VI, cap. VIII.

57 Vid., por ejemplo, Inv. V, §9 (A 345). 
de representaciones y de juicios dotados de validez objetiva; y no están dados más que como objetivos de vivencias intencionales del yo." ${ }^{58} \mathrm{Se}$ trata de un supuesto no fundamentado; de una convicción que supongo que debe ser puesta en la cuenta terrible del sentido común. Gierto; pero, ¿cuál es su suelo teórico?

La respuesta está, sin duda, en aquello que constituye la unidad intrínseca y autónoma del yo reducido, incluso por debajo de la unidad mundanal del yo empírico: el tiempo. El tiempo reducido es el mismo tiempo real. Si Husserl abandonaba esta tesis, el realismo, en los términos de sentido común en que lo aceptaban las Investigaciones, se venía irremediablemente abajo. Ahora bien, el tiempo fenomenológico es inmanente, y el tiempo real es, tan sólo, objeto de escorzo en aquél. El problema de la acogida en los mismos árboles porfirianos tanto de las propiedades objetivas trascendentes como de las "sensaciones" inmanentes se repite en este punto delicado; o, más bien, llega a su máximo, si tenemos en cuenta que el tiempo es la forma del ser real, y no algo así como un accidente de algunos seres reales. Es perfectamente comprensible que la profundización en las dificultades de la conciencia del tiempo deparara la ocasión para el pensamiento de la reducción fenomenológico-trascendental.

Pero veamos todavía nosotros las posibilidades y las oscuridades que dejaba abiertas el texto antes citado, el único en todas las Investigaciones, que tiene por tema la "conciencia subjetiva del tiempo". No hemos examinado hasta el final 1) por qué se emplea en él el término "sensación" y 2) cuál es la paradoja que aparece aludida.

Las dos preguntas tienen respuesta en el mismo dato: el flujo continuo a través del ahora inmóvil se expone a sí mismo, como si se tratara de sensaciones que fueran autointencionales y que, a un tiempo, escorzaran un objeto sin ayuda de ninguna "aprehensión". Pues, si meditamos sobre el modo de presentársenos el flujo temporal fenomenológico, caemos en la cuenta de que es absurdo admitir que se nos da como el objeto constituido ante nuestra conciencia por ciertas vivencias ajenas a él (o sea, que ni están en él, ni son él mismo). Tales vivencias se encontrarán siempre en el tiempo fenomenológico, y se presentarán, en el instante mismo en que son vividas, como partes fluyentes de la conciencia. $Y$, además, es asimismo absurdo postular alguna aprehensión que ayudara a constituir el tiempo inmanente, ya que él no es concepto, sino sucesión pura (y el problema no es ahora cómo se construye la representación conceptual del tiempo, sino cómo se vive inmediatamente el fluir del tiempo).

Para la captación originaria del tiempo no valen, pues, las nociones 58 Inv. V, \&7 (A 337). 
de 'fenómeno', 'sensación' o 'contenido primario' y 'aprehensión'; aunque la que más próxima está al dato es la de 'sensación'. ${ }^{59}$ Como he escrito antes, hay aquí algo así como sensaciones fluyentes, que se presentan a sí mismas a título de pura sucesión dispuesta a estar llena con cualquier materia consciente; y esas sensaciones, que son el tiempo inmanente mismo, escorzan el tiempo espacializado y trascendente que apoya la idea de causa y ser real. O bien el tiempo fenomenológico se da ante unas "vivencias" absolutamente no susceptibles de ser percibidas internamente (ni siquiera en una percepción interna inadecuada), que, por principio, ya no están en el tiempo (de modo que el estrato básico de la conciencia es inconsciente y atemporal); o bien la conciencia, en su fondo mismo, es tiempo, y el tiempo es la conciencia (y el ser radical es tiempo).

59 Cfr. Vorlesungen zur Phänomenologie des inneren Zeitbewußstseins (1905), en: Hua. X (Ed. R. Boehm) (Den Haag 1966). Ya desde el $\S 1$ se plantea el problema esencial en estos términos. 